\title{
AUQ-QUA 2012
}

\section{Sessions scientifiques XII, XIII, XIV Samedi (p.m.), le 10 novembre 2012}

Les sessions scientifiques suivantes ont été rendues possibles grace à la contribution non restrictive de nos partenaires :

\author{
Abbott Laboratories Ltd. \\ Amgen Canada \\ Astellas Pharma Canada Inc. \\ AstraZeneca \\ Janssen Inc. \\ GlaxoSmithKline Inc \\ Pfizer Canada \\ Sanofi-Aventis \\ Watson Pharma Company Canada
}

PARTENAIRES ELITES/ELITE SPONSORS

PARTENAIRES MAJEURS/MAJOR SPONSORS

Eli Lilly Canada Inc.

PARTENAIRES COLLABORATEURS/ASSOCIATE SPONSORS Allergan Inc.

Medtronic of Canada

\section{Session scientifique XII}

Objectifs éducatifs : À la fin de cette conférence, l'auditoire apprendra les défis que représente une vie active complète pour homme remarquable souffrant d'un handicap physique.

\section{2 h 00 - 13 h 00}

Lunch conférence

Salon Jacques-Cartier

Conférencier : M. Dean Bergeron

Directeur Marketing et promotion de la santé, La Capitale assurances et gestion du patrimoine Inc.

Modérateur : Stéphane Bolduc

\section{Session scientifique XIII}

Objectifs éducatifs : À la fin de cette session, les participants decouvriront les importants développements dans les chirurgies endourologiques. Ils possèderont mieux les aspects techniques et les implications cliniques, de même que les limites de ces approches.

\section{3 h 15 - 13 h 50}

Quoi de neuf en endourologie

Conférencier : Olivier Traxer

Hopital Tenon, Paris, France

Modérateur : Luc Valiquette

\section{Session scientifique XIV}

Objectifs éducatifs : À la fin de cette session, les participants comprendront davantage les diverses hypothèses étiologiques actuelles sur l'infertilité chez I'homme. Une mise a jour sur les nouvelles démarches diagnostiques et thérapeutiques seront revisées.

14 h 00 - 14 h 30

L'épididyme et l'étiologie de l'infertilité : L'homme vasovasostomisé comme modèle

Conférencier: Robert Sullivan

Centre de recherche du CHUQ

Modérateur : Francois Benard 